\title{
LOCALLY NILPOTENT GROUPS WITH THE MAXIMUM CONDITION ON NON-NILPOTENT SUBGROUPS
}

\author{
MARTYN R. DIXON \\ Department of Mathematics, University of Alabama, Tuscaloosa, AL 35487-0350, USA \\ e-mail:mdixon@gp.as.ua.edu \\ and LEONID A. KURDACHENKO \\ Department of Algebra, University of Dnepropetrovsk, Provulok Naukovyj 13, Dnepropetrovsk 50, \\ 320625 DSP, Ukraine \\ e-mail:mmf@ff.dsu.dp.ua
}

(Received 10 May, 1999)

\begin{abstract}
In this paper the authors consider the class of locally nilpotent groups that have the maximum condition on non-nilpotent subgroups.
\end{abstract}

1991 Mathematics Subject Classification. 20F19, 20 E07.

1. Introduction. The class of groups in which every proper subgroup is nilpotent has been considered in several papers. Finite groups with all proper subgroups nilpotent were considered by $\mathrm{O}$. Yu. Schmidt in [16], where he showed that such groups are always soluble. Subsequent papers on the subject include [14] and [17]. Such groups are, of course, locally nilpotent or finitely generated. In either case the structure of groups with all proper subgroups nilpotent can be very complicated, as is seen from the Heineken-Mohamed examples. (See, for example, [6], [4], [13].)

In [17], H. Smith also considered the class of locally nilpotent groups in which each group satisfied a certain finiteness condition on its non-nilpotent subgroups. We continue this theme in the current paper. A group $G$ is said to satisfy max-(nonnil), the maximum condition on non-nilpotent subgroups, if every non-empty set of non-nilpotent subgroups has a maximal element, or equivalently, if every ascending chain of non-nilpotent subgroups terminates in finitely many steps. The class of groups with the maximum condition on non-abelian subgroups was considered in [12], where a complete structure theorem was given. In [17], Smith proved that a torsionfree locally nilpotent group with max-(non-nil) is necessarily nilpotent; (in fact Smith's result had apparently weaker hypotheses than max-(non-nil)). In this paper we consider further the class of groups with max-(non-nil). It is easy to construct locally nilpotent groups with max-(non-nil) in which not every proper subgroup is nilpotent; for example the direct product of a Heineken-Mohamed type group with a finite nilpotent group will do. (Indeed, it is not difficult to show that an infinite locally finite, non-nilpotent group with max-(non-nil) is a direct product of finitely many Sylow $p$-subgroups, exactly one of which is infinite and also a locally nilpotent non-nilpotent group). As further motivation for our work we note that Smith also proved in [17] that if $p$ is a prime then a locally nilpotent $p$-group $G$ with max-(non-nil) is nilpotent provided $G / G^{\widetilde{\mho}}$ is infinite; here $G^{\widetilde{\wp}}$ denotes the finite residual of $G$. In our work the finite residual again plays an important role.

We cannot obtain a detailed description of locally nilpotent groups with max(non-nil), because of examples such as those of Heineken-Mohamed type. Our results divide naturally into two cases. In Section 2 we give a number of preliminary 
results on groups with max-(non-nil). In Section 3 we show that if $G$ is a non-nilpotent, locally nilpotent group with max-(non-nil) and if $T$ is the torsion subgroup of $G$ then $G^{\mathfrak{\mho}} \leq T$. Our main result in Section 3 (Theorem A) partially generalizes Theorem 2.2 of [17]. We consider the case in which $G / G^{\mathfrak{s}}$ is not finitely generated and $G$ is non-nilpotent. We prove, among other things, that in this case $T / G^{\widetilde{\mho}}$ is finite and $G / T$ is a nilpotent minimax group.

In Section 4 we consider the much harder case, in which $G / G^{\mathfrak{\mho}}$ is finitely generated. In this case $G^{\widetilde{\Im}}$ need not be nilpotent. We show that $G^{\mathfrak{\wp}}$ is $\widetilde{\wp}$-perfect if $G$ is nonnilpotent; in the case of locally nilpotent $p$-groups this is an immediate corollary of Theorem 2.3 of [17]. In Theorem B we indicate that if $G$ is non-nilpotent, but $G^{\mathfrak{\jmath}}$ is nilpotent, and if $G / G^{\mathfrak{\wp}}$ is finitely generated then the structure of $G^{\mathfrak{\wp}}$ is very restricted,

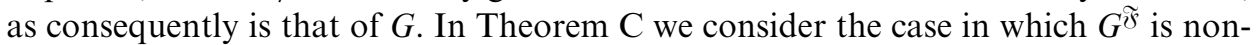
nilpotent. In this case $G^{\widetilde{\mho}}$ is a $p$-group, for some prime $p$, and every proper subgroup of $G^{\mathscr{\mho}}$ is nilpotent.

Our notation, when not explained, is that in standard use. We should like to thank Professor A. O. Asar for sending us a preprint of his paper [1] and also the referee for his numerous suggestions which improved this paper.

2. Preliminaries. In this section we collect some easy preliminary results together that we use throughout the paper. The first two of these are quite straightforward and so we omit their proof. As usual we let max denote the maximum condition on subgroups.

LEMMA 2.1. Let $G$ be a group satisfying max-(non-nil).

(i) If $H$ is a subgroup of $G$, then $H$ satisfies max-(non-nil).

(ii) If $H$ is a normal subgroup of $G$, then $G / H$ satisfies max-(non-nil).

The next result uses the well-known fact that a soluble group with the maximum condition on subgroups is polycyclic.

Lemma 2.2. Let $G$ be a group satisfying max-(non-nil), and suppose $H$ is a nonnilpotent subgroup of $G$. Then $N_{G}(H) / H$ satisfies max. In particular, if $N_{G}(H)$ is locally (soluble-by-finite), then $N_{G}(H) / H$ is polycyclic-by-finite.

Lemma 2.3. Let $G$ be a group satisfying max-(non-nil). Suppose that $H, A, B$ are subgroups of $G$ such that $A \triangleleft B, A$ and $B$ are $H$-invariant, and $H \cap B \leq A$. If $A H$ is non-nilpotent, then $B / A$ satisfies the maximal condition on $H$-invariant subgroups.

Proof. Suppose that $B_{1} / A \leq B_{2} / A \ldots$ is an ascending chain of $H$-invariant subgroups of $B / A$. Then there is an integer $k$ such that $B_{n} H=B_{k} H$, for all $n \geq k$. Since $H \cap B \leq A$ we have $B_{n}=B_{k}$, by the Dedekind law.

It is clear that any strictly ascending infinite chain of subgroups in a group with max-(non-nil) has the property that every subgroup in the chain is nilpotent. We record this in our next lemma.

Lemma 2.4. Let $G$ be a group satisfying max-(non-nil) and suppose that $G_{1} \leq G_{2} \leq \ldots \leq G_{n} \leq \ldots$ is a strictly ascending chain of subgroups. Then $G_{n}$ is nilpotent, for each $n \in \mathbb{N}$. 
There are some natural, simple consequences of these easy results that will be very useful in what follows. First we have the following result.

Corollary 2.5. Let $G$ be a group satisfying max-(non-nil). Suppose that $H, K$ are subgroups of $G$ such that $K \triangleleft H$. If $H / K=A / K \times B / K$ and $A / K, B / K$ do not satisfy max, then $H$ is nilpotent.

Proof. Since $H / B \cong A / K$ we see that $H / B$ does not satisfy max. By Lemma 2.2 the subgroup $B$ is nilpotent. Similarly the subgroup $A$ is nilpotent. By Fitting's Theorem $H=A B$ is also nilpotent.

Corollary 2.6. Let $G$ be a group satisfying max-(non-nil). Suppose that $H, K$ are subgroups of $G$ such that $K \triangleleft H$. If $H / K=\operatorname{Dr}_{\lambda \in \Lambda} A_{\lambda} / K$ is a direct product of infinitely many non-trivial groups, then $H$ is nilpotent.

Proof. Since $\Lambda$ is infinite it contains infinite subsets $\Lambda_{1}, \Lambda_{2}$ such that $\Lambda_{1} \cup \Lambda_{2}=$ $\Lambda, \Lambda_{1} \cap \Lambda_{2}=\emptyset$. Now put $A / K=\operatorname{Dr}_{\lambda \in \Lambda_{1}} A_{\lambda} / K, B / K=\operatorname{Dr}_{\lambda \in \Lambda_{2}} A_{\lambda} / K$. Clearly, both $A / K$ and $B / K$ do not satisfy max and we can apply Corollary 2.5 .

Lemma 2.7. Let $G$ be a group satisfying max-(non-nil). Suppose that $H, K, S$ are subgroups of $G$ such that $K \triangleleft H$. Suppose also that

(i) $H / K=\operatorname{Dr}_{\lambda \in \Lambda} A_{\lambda} / K$, where $A_{\lambda} \neq K$ for every $\lambda \in \Lambda$ and the set $\Lambda$ is infinite;

(ii) $A_{\lambda}$ is $S$-invariant for every $\lambda \in \Lambda$;

(iii) $H \cap S \leq K$.

Then the subgroup HS is nilpotent.

Proof. Write $H / K=U / K \times V / K$, where each of $U / K$ and $V / K$ is a direct product modulo $K$ of infinitely many $A_{\lambda}$ 's. There is an infinite strictly ascending chain from $U S$ to $H S$ and so $U S$ is nilpotent, as is $V S$. Thus there is an integer $k$ such that $\left[U,_{k} S\right] \leq S$ and $\left[V,{ }_{k} S\right] \leq S$. It is easy to see that, by induction, $\left[H,,_{k} S\right] \leq K S$ and, since $K S$ is nilpotent, it follows that $S$ is subnormal in $H S$. Since $H, S$ are nilpotent it follows that $H S$ is also nilpotent, as required.

3. The case in which $G / G^{\widetilde{\mho}}$ is not finitely generated. In this section we consider the case of a locally nilpotent group $G$ such that $G / G^{\mathfrak{\wp}}$ is not finitely generated. We shall require some terminology which is well known, but which we briefly explain. Let $G$ be an abelian group of finite (special) rank, and let $H$ be a finitely generated subgroup of $G$ such that $G / H$ is periodic. Let $\operatorname{Sp}(G)$, the Spectrum of $G$, denote the set of primes $p$ such that a Sylow $p$-subgroup of $G / H$ is infinite.

If $L$ is another finitely generated subgroup of $G$ such that $G / L$ is periodic, then both factors $H /(H \cap L)$ and $L /(H \cap L)$ are finite. It follows that the set $S p(G)$ is independent of the choice of finitely generated subgroup $H$.

Now let $G$ be a nilpotent group of finite rank and let

$$
1=G_{0} \leq G_{1} \leq \ldots \leq G_{n}=G
$$

be a central series of $G$. We let 


$$
\operatorname{Sp}(G)=\operatorname{Sp}\left(G_{1}\right) \cup \operatorname{Sp}\left(G_{2} / G_{1}\right) \cup \ldots \cup \operatorname{Sp}\left(G_{n} / G_{n-1}\right) .
$$

Clearly the set $\operatorname{Sp}(G)$ is independent of the choice of central series.

With these preliminaries out of the way we can prove a crucial lemma.

Lemma 3.1. Let $G$ be a locally nilpotent group satisfying max-(non-nil) and let $T$ be the torsion subgroup of $G$. If $G$ is non-nilpotent, then $G / T$ is either finitely generated or nilpotent minimax with $\operatorname{Sp}(G / T)=\{p\}$, for some prime $p$. Moreover, in the latter case, $G$ contains a normal nilpotent subgroup $U$ such that $G / U$ is a Prüfer p-group.

Proof. We may assume that $G / T$ is non-trivial. By Theorem 2.2 of [17] $G / T$ is nilpotent. In particular, $G \neq G^{\prime}$. Let $H=G / G^{\prime}$. Let $P$ be the torsion subgroup of $H$ and let $K=H / P$. Note that if $H / H^{n}$ is infinite, for some natural number $n$, then $H / H^{n}$ is a bounded abelian group and hence is a direct sum of infinitely many nontrivial cyclic groups. Also the same is true of $G / G^{\prime} G^{n}$. It follows from Corollary 2.6 that $G$ is then nilpotent, a contradiction. Thus $H / H^{n}$ is finite for each $n$.

Suppose first that $K$ has infinite rank. Let $\mathcal{M}$ be a maximal linearly independent subset of $K$ and let $M=\langle\mathcal{M}\rangle$. Then $M=\operatorname{Dr}_{i \in I} N_{i}$, where $N_{i} \cong \mathbb{Z}$. Clearly $K / M$ is periodic and $I$ is infinite. Let $J$ be a countably infinite subset of $I$ and let $\left\{p_{j} \mid j \in J\right\}$ be an infinite set of primes. Let $L=\operatorname{Dr}_{i \in I J} N_{i} \times \operatorname{Dr}_{j \in J} N_{j}^{p_{j}}$. Then $K / L$ is periodic and $\pi(K / L)$ is infinite. Thus $K / L=\operatorname{Dr}_{p \in \mathbb{P}} X_{p} / L$, where for infinitely many primes $p$ we have $X_{p} \neq L$. By Corollary 2.6 it follows that $G$ is nilpotent in this case, which is a contradiction. Consequently $H / P$ has finite rank.

Suppose next that $P$ is trivial. Let $\left\{h_{1}, \ldots, h_{r}\right\}$ be a maximal independent subset of $H$, and let $R=\left\langle h_{1}, \ldots, h_{r}\right\rangle$. Then $H / R$ is a periodic group. If $H / R$ is finite, then $H$ is finitely generated. If $H / R$ is infinite then, by Corollary $2.5, H / R=H_{1} / R \times H_{2} / R$ where $H_{1} / R$ is a Prüfer p-subgroup, for some prime $p$, and $H_{2} / R$ is finite. It follows that $H$ is minimax and $S p(H)=\{p\}$. Moreover in this case $H / H_{2} \cong C_{p^{\infty}}$. Let $U$ be a preimage of $\mathrm{H}_{2}$ in $G$. By Lemma 2.4, $U$ is nilpotent.

Suppose that $P \neq 1$. If $K=H / P$ is not finitely generated let $\left\{a_{1}+P, \ldots, a_{n}+P\right\}$ be a maximal linearly independent subset of $K$. If $M=\left\langle a_{1}, \ldots, a_{n}\right\rangle$ then $P \cap M$ is finite so that if $\pi(P)$ is infinite then $\pi(H / M)$ is also infinite and we deduce that $G$ is nilpotent, using Corollary 2.6. Consequently $\pi(P)$ is finite.

Let $P=\underset{q \in \pi(P)}{\operatorname{Dr}} S_{q}$, where $S_{q}$, is a Sylow $q$-subgroup of $P$. Since $S_{q}$ is a pure subgroup of $H$ and $H / H^{n}$ is finite for each natural number $n$, it follows that $S_{q} / S_{q}^{n}$ is also finite, by [9, Lemma 6], and Lemma 7 of [9] then shows that $S_{q}=X_{q} \times Y_{q}$, where $X_{q}$ is finite and $Y_{q}$ is a divisible subgroup. Since $H=Y_{q} \times W_{q}$ for some subgroup $W_{q}, H / W_{q} \cong Y_{q}$ and Corollary 2.6 yields that $Y_{q}$ is either trivial or a Prüfer $q$ subgroup, for every $q \in \pi(P)$. If we assume that $Y_{q} \neq 1$ for some $q$ then, since $H / P$ is of finite rank but not finitely generated, $H$ has a factor group which is the direct product of two Prüfer subgroups. Corollary 2.6 again shows that in this case $G$ is nilpotent. This contradiction proves that $Y_{q}=1$ for all $q \in \pi(P)$ and hence $P$ is finite. Then $H$ has finite rank and as above we can show $H$ is minimax and that $G$ contains a nilpotent subgroup $U$ such that $G / U \cong C_{p^{\infty}}$.

If $H / P$ is finitely generated, then $H=P \times Q$, for some finitely generated torsionfree abelian group $Q$ and Corollary 2.6 implies that $\pi(P)$ is finite. Similar arguments to those above show that either $P=E \times F$, where $E$ is a Prüfer $p$-subgroup for some prime $p$ and $F$ is finite, or $P$ is finite. Hence either $H$ is finitely generated or 
$H$ has finite rank. As above $H$ is minimax and we can construct a normal nilpotent subgroup $U$ of $G$ such that $G / U \cong C_{p^{\infty}}$. Hence in every case either $G / T G^{\prime}$ is finitely generated or $G / T G^{\prime}$ is minimax with $\operatorname{Sp}\left(G / T G^{\prime}\right)=\{p\}$. By [15, Corollary to Theorem 2.26] it follows that either $G / T$ is finitely generated or $G / T$ is minimax with $S p(G / T)=\{p\}$.

The following corollary is immediate from Lemma 3.1 and the fact that a torsionfree nilpotent minimax group is residually finite [15, Theorem 9.38].

COROLlary 3.2. Let $G$ be a locally nilpotent group satisfying max-(non-nil) and suppose that $G^{\widetilde{\Im}}$ is the finite residual of $G$. If $G$ is non-nilpotent, then $G^{\widetilde{\mho}}$ is periodic.

Next we prove that the torsion subgroup of our group $G / G^{\mathfrak{\wp}}$ is quite restricted. First we prove the following result.

Lemma 3.3. Let $G$ be a locally nilpotent group satisfying max-(non-nil). Suppose

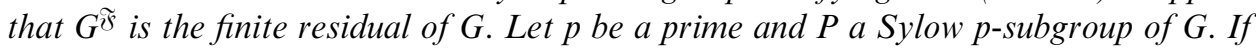
$G$ is non-nilpotent, then $P /\left(P \cap G^{\widetilde{\mho}}\right)$ is finite.

Proof. Let $H=G^{\mathfrak{\Im}}$, and suppose that $1 \neq x \in P \backslash H$. Then there is a normal subgroup $G_{x}$ of finite index such that $x \notin G_{x}$. We have $G / G_{x}=U_{x} / G_{x} \times V_{x} / G_{x}$, where $U_{x} / G_{x}$ is a Sylow $p$-subgroup of $G / G_{x}, V_{x} / G_{x}$ is a Sylow $p^{\prime}$-subgroup of $G / G_{x}$. Since $x$ is a $p$-element, $x \notin V_{x}$ and $G / V_{x}$ is a finite $p$-group. Thus we can assume that $G / G_{x}$ is a $p$-group. Let

$$
\mathcal{S}=\{S \mid S \text { is a normal subgroup of } G \text { such that } G / S \text { is a finite } p \text {-group }\}
$$

and

$$
M=\bigcap\{S: S \in \mathcal{S}\}
$$

The argument above shows that $M \cap P=H \cap P$. Set $D=G / M$. By Corollary 2.6 $D / D^{\prime} D^{p}$ is finite, so that Lemma 2 of [7] implies that the torsion subgroup of $D$ is finite. In particular, $P M / M \cong P /(P \cap M)$ is finite.

We require a technical result that will be needed in our proof that the torsion subgroup of $G / G^{\widetilde{\mho}}$ is finite. This in turn requires several small lemmas, which are probably well known, but we include them here.

Lemma 3.4. Let $G$ be a nilpotent group. Suppose that $A$ is a normal infinite $\sigma$ subgroup of $G$, for some set of primes $\sigma$, such that $G / A$ is finitely generated. Then there is a normal subgroup $M$ of $G$ such that $G / M$ is an infinite $\sigma$-group, and if $A \leq Z(G)$, then we can choose $M$ to be finitely generated.

Proof. Suppose first that $A \leq Z(G)$. There exists $M \leq G$, with $M$ finitely generated, such that $G=M A$. Clearly $M \triangleleft G$ and, since $M$ is finitely generated, $A \cap M$ is finite. Hence $G / M$ is of the desired type in this case. Suppose that $G$ is nilpotent of class $c$. If $A$ is not necessarily central then, since $G / \gamma_{c}(G)$ is not finitely generated, $A \gamma_{c}(G) / \gamma_{c}(G)$ is an infinite $\sigma$-subgroup with $G / A \gamma_{c}(G)$ finitely generated and so, 
inductively, we may assume that $G / \gamma_{c}(G)$ contains a normal subgroup $M / \gamma_{c}(G)$ such that $G / M$ is an infinite $\sigma$-group. This completes the proof.

Lemma 3.5. Let $G$ be a nilpotent group and suppose that $H$ is a normal abelian subgroup of $G$ such that $G / H$ is a $\pi$-group, for some set of primes $\pi$. If $K \leq H$, then $[K, G]$ is a $\pi$-group.

Proof. Let $\left[K,{ }_{i} G\right]=[K, \underbrace{G, \ldots, G}_{i}]$. We prove that, for each $i \geq 1,\left[K,{ }_{i} G\right] /\left[K,{ }_{i+1} G\right]$ is a $\pi$-group; then since $G$ is nilpotent we have $\left[K,{ }_{c} G\right]=1$, for some $c$, and the claim follows.

Let $x \in\left[K,{ }_{i-1} G\right], g \in G$. Then there exists a $\pi$-number $n$ such that $g^{n} \in H$ and so $\left[x, g^{n}\right]=1$, since $H$ is abelian and normal in $G$. Using commutator calculus we see that

$$
1=\left[x, g^{n}\right]=[x, g]^{n} \bmod \left[K,{ }_{i+1} G\right]
$$

so that the claim follows.

Lemma 3.6. Let $G$ be a nilpotent group and suppose that $M \triangleleft N \triangleleft G$. Suppose that for some set of primes $\pi, G / N$ is an infinite $\pi$-group and $N / M$ is an infinite $\pi^{\prime}$-group. Then $G$ has a normal subgroup $K$ such that $G / K$ is periodic and the direct product of an infinite $\pi$ - and an infinite $\pi^{\prime}$-group.

Proof. Consider $M^{G}=M[M, G]$ and apply Lemma 3.5 to the group $G / N^{\prime}$. We have that

$$
[M, G] N^{\prime} / N^{\prime}=\left[M N^{\prime} / N^{\prime}, G / N^{\prime}\right]
$$

is a $\pi$-group. However $[M, G] M N^{\prime} / M N^{\prime}$ is an image of this and so is also a $\pi$-group. Thus $M^{G} N^{\prime} / M N^{\prime}$ is a $\pi$-group. However $N / M$ is a $\pi^{\prime}$-group, so that $N / M N^{\prime}$ is also $\pi^{\prime}$ and, since $M^{G} N^{\prime} / M N^{\prime} \leq N / M N^{\prime}$, we have $M^{G} \leq M^{G} N^{\prime}=M N^{\prime}$. Since $G$ is nilpotent and $N / M$ is infinite, it follows that $N / M N^{\prime}$ is infinite and hence so is $N / M^{G}$. Thus $G / M^{G}$ has the required properties.

Lemma 3.7. Suppose that $G$ is a nilpotent group with torsion subgroup $T$ satisfying

(i) $T$ is an infinite $\pi$-group, for some set of primes $\pi$;

(ii) $G / T$ has finite rank;

(iii) $G$ has a series $1 \leq T=Z_{0} \leq X_{1} \leq Z_{1} \leq X_{2} \leq Z_{2} \leq \ldots \leq X_{c} \leq Z_{c}=G$, where $\left\{Z_{i} / T\right\}$ is the upper central series of $G / T, X_{i+1} / Z_{i}$ is finitely generated and $Z_{i} / X_{i}$ is either trivial or an infinite periodic group, and at least one of these factors contains an infinite $\pi^{\prime}$-subgroup.

Then $G$ has a periodic factor group $G / H$ such that the Sylow $\pi$ and Sylow $\pi^{\prime}-$ subgroups of $G / H$ are infinite.

Proof. Note that by changing the $X_{i}$ accordingly, we may always suppose that the Sylow $\pi$ - (respectively $\pi^{\prime}$-) subgroup of $Z_{i} / X_{i}$ is trivial or infinite. We let

$$
1 \leq T=Z_{0} \leq X_{1} \leq Z_{1} \leq X_{2} \leq Z_{2} \leq \ldots \leq X_{c} \leq Z_{c}=G
$$

be a series as in the statement of the lemma. 
We use induction on the total number of infinite periodic factors in $G / T$ in the above series. By assumption at least one of the factors is infinite. If there is only one, say $Z_{k} / X_{k}$, then $G / Z_{k}$ is finitely generated and the result follows if $Z_{k} / X_{k}$ has both an infinite $\pi$ - and infinite $\pi^{\prime}$-subgroup, using Lemma 3.4 applied to $G / X_{k}$. Thus we may assume that $Z_{k} / X_{k}$ is an infinite $\pi^{\prime}$-group. Then, according to Lemma 3.4 applied to appropriate images of $G / X_{k}$, there is a finitely generated normal subgroup $M / X_{k}$ such that $G / M$ is an infinite $\pi^{\prime}$-group. Then $M / T$ is finitely generated and, again by Lemma 3.4, $M$ contains a normal subgroup $L$ such that $M / L$ is an infinite $\pi$-group. Then, by Lemma 3.6, the result holds for $G$.

Consequently in the series (1) we may assume that at least two of the factors $Z_{i} / X_{i}$ are infinite. Let $k$ be largest such that $Z_{k} / X_{k}$ is infinite. Then $G / Z_{k}$ is finitely generated. If $Z_{k} / X_{k}$ has both an infinite $\pi$ - and an infinite $\pi^{\prime}$-factor, then we apply Lemma 3.4 to $G / X_{k}$ and deduce the result.

If $Z_{k} / X_{k}$ is an infinite $\pi$-group then, by hypothesis, there exists $l<k$ such that $Z_{l} / X_{l}$ has an infinite $\pi^{\prime}$-factor. If $Z_{l} / X_{l}=A / X_{l} \times B / X_{l}$, where $A / X_{l}$ is an infinite $\pi^{\prime}$ group, then we apply the induction hypothesis to the group $G / B$, (interchanging the roles of the sets of $\pi$ and $\pi^{\prime}$ ) and hence obtain the result for $G$. If $Z_{k} / X_{k}$ is an infinite $\pi^{\prime}$-group and for some $l$ we have $Z_{l} / X_{l}$ has an infinite $\pi$-factor, then the same argument proves the result again. Thus we may assume that all infinite factors $Z_{i} / X_{i}$ are $\pi^{\prime}$-groups. But then applying Lemma 3.4 to $G / X_{k}$ gives us a finitely generated normal subgroup $M / X_{k}$ such that $G / M$ is an infinite $\pi^{\prime}$-group. Then applying the induction hypothesis to $M$ yields that $M$ has a factor group $M / L$ which is the direct product of an infinite $\pi$-group $R / L$ with an infinite $\pi^{\prime}$-group $S / L$. We have $S \triangleleft M \triangleleft G$ with $M / S$ an infinite $\pi$-group and $G / M$ an infinite $\pi^{\prime}$-group. Now apply Lemma 3.6. The result follows.

With this digression out of the way we now have the following result.

Proposition 3.8. Let $G$ be a locally nilpotent group satisfying max-(non-nil). Let $T$ be the torsion subgroup of $G$ and $G^{\mathfrak{\mho}}$ the finite residual of $G$. If $G$ is non-nilpotent and $G / T$ is not finitely generated, then $T / G^{\widetilde{\Im}}$ is finite. In particular, $G / G^{\widetilde{\mho}}$ is a nilpotent minimax group with finite torsion subgroup.

Proof. By Corollary 3.2, $G^{\widetilde{s}} \leq T$. Suppose, to the contrary, that $T / G^{\widetilde{s}}$ is infinite. Let $q$ be a prime. Then if $S_{q}$ is a Sylow $q$-subgroup of $G$ it follows that $S_{q} G^{\widetilde{\wp}} / G^{\widetilde{\wp}}$ is finite, by Lemma 3.3, and hence $\pi=\pi\left(T / G^{\mathscr{\mho}}\right)$ is infinite. By Lemma 3.1, $S p(G / T)=\{p\}$, for some prime $p$. For every prime $q \in \pi$ with $q \neq p$ choose a maximal $G$-invariant subgroup $M_{q} \leq S_{q}$ such that $S_{q} \cap G^{\mathfrak{\wp}} \leq M_{q}$. Set

$$
M=\left(\operatorname{Dr}_{p \neq q \in \pi} M_{q}\right) \times\left(\underset{q \notin \pi}{\operatorname{Dr}} S_{q}\right) \times S_{p} .
$$

The factors $S_{q} / M_{q}$ for $q \neq p$ are $G$-chief factors and are therefore central; (see, for example, [15, Corollary 1 to Theorem 5.27]). It follows that $T / M \leq Z(G / M)$ and so $G / M$ is nilpotent. Now we can apply Lemma 3.7 to the group $G / M$ and deduce that $G$ contains a normal subgroup $H$ such that $G / H$ is periodic and the Sylow $p$-subgroup and Sylow $p^{\prime}$-subgroup of $G / H$ are infinite. Corollary 2.5 then shows that $G$ is nilpotent, a contradiction which proves that $T / G^{\mathfrak{\mho}}$ is finite.

Next, we obtain more information concerning $G^{\Re}$. 
Proposition 3.9. Let $G$ be a locally nilpotent group satisfying max-(non-nil). Let $T$ be the torsion subgroup of $G$ and $G^{\mathfrak{\mho}}$ the finite residual of $G$. Suppose that $G$ is nonnilpotent and $G / T$ is not finitely generated. Then $G^{\widetilde{\wp}}$ is a p-group, where $p=\operatorname{Sp}(G / T)$.

Proof. By Lemma 3.1, $G$ contains a nilpotent, normal subgroup $U \geq T$ such that $G / U$ is a Prüfer $p$-group, for some prime $p$. By Proposition 3.8, $G / G^{\widetilde{\mho}}$ is nilpotent and minimax. If $H$ is a $G$-invariant subgroup of $G^{\mathfrak{\mho}}$ such that $G^{\mathfrak{\mho}} / H$ is finite, then $G / H$ is residually finite; (see, for example, [15, Theorem 9.38]). This contradiction shows that $G^{\widetilde{\mho}}$ contains no proper $G$-invariant subgroups of finite index. Suppose that $G^{\widetilde{\wp}}$ is not a $p$-group. Then the observation above implies that the Sylow $p^{\prime}-$ subgroup of $G^{\widetilde{\mho}}$ is infinite. It follows that the Sylow $p^{\prime}$-subgroup of $T$ is infinite. Let $T=P \times Q$, where $P$ (respectively $Q$ ) is a Sylow $p$-subgroup (respectively Sylow $p^{\prime}$ subgroup) of $T$. We first suppose that $P=1$. Since $U$ is nilpotent and normal in $G, T$ has a finite $U$-central series

$$
1=T_{0} \leq T_{1} \leq \ldots \leq T_{n}=T,
$$

the terms of which are normal in $G$.

Let $x \in G$, and let $V=\langle U, x\rangle$. Since $G / V$ is a Prüfer $p$-group, $V$ is nilpotent, by Lemma 2.4. Let

$$
1=Y_{0} \leq Y_{1} \leq \ldots \leq Y_{m}=T_{1}
$$

be the upper $V$-central series of $T_{1}$. Suppose that $Y_{2} \neq Y_{1}$. If $y \in Y_{2} \backslash Y_{1}$, then $\left[T_{1}, U\right]=1$ implies $1 \neq[y, x] \in Y_{1}$, so $y^{x}=y y_{1}$, for some element $y_{1} \in Y_{1}$. Let $k=|x U|, q=\left|y_{1}\right|$, so that $(k, q)=1$. We have $y=x^{-k} y x^{k}=y y_{1}^{k}$; thus $y_{1}^{k}=1$. Since $y_{1}^{q}=1$ it follows that $y_{1}=1$. This means that $Y_{1}=T_{1}$ and hence, by induction, $\left\{T_{i} \mid 0 \leq i \leq n\right\}$ is also a $V$-central series. Since $x$ is an arbitrary element of $G$, $\left\{T_{i} \mid 0 \leq i \leq n\right\}$ is therefore a $G$-central series. Since $G / T$ is nilpotent it follows that $G$ is also nilpotent. In the general case, when $P \neq 1$, the argument above shows that $G / P$ is nilpotent. By Lemma 3.7 applied to $G / P$, we see that there exists a normal subgroup $S \geq P$ such that $G / S$ is periodic and the Sylow $p$-subgroup and Sylow $p^{\prime}-$ subgroup of $G / S$ are infinite. This is a contradiction, since Corollary 2.5 then implies that $G$ is nilpotent. Hence $G^{\widetilde{\mho}}$ is a $p$-group, as required.

We have now obtained rather a lot of information concerning a locally nilpotent group $G$ with max-(non-nil) in the case in which $G / G^{\widetilde{\Im}}$ is not finitely generated. We summarize our results as Theorem A.

Theorem A. Let $G$ be a locally nilpotent group satisfying max-(non-nil). Let $T$ be the torsion subgroup of $G$, and let $G^{\widetilde{\mho}}$ be the finite residual of $G$. If $G$ is non-nilpotent and $G / G^{\widetilde{\mho}}$ is not finitely generated then the following hold.

(1) $G^{\mathfrak{\wp}} \leq T$;

(2) $T / G^{\mathfrak{\Im}}$ is finite;

(3) $G / T$ is a nilpotent minimax group;

(4) $\operatorname{Sp}(G / T)=\{p\}$, for some prime $p$;

(5) $G^{\widetilde{\aleph}}$ is a p-subgroup;

(6) $G$ contains a normal nilpotent subgroup $U$ such that $G / U$ is a Prüfer p-group;

(7) if $S$ is a non-nilpotent subgroup of $G$, then $G=S U$. 
Proof. First note that (1) follows from Corollary 3.2. Next we note that if $G / G^{\dddot{s}}$ is not finitely generated then $G / T$ is not finitely generated either. For, suppose that $G / T$ is finitely generated. Then $T / G^{\widetilde{\wp}}=\operatorname{Dr}_{\lambda \in \Lambda}\left(P_{\lambda} / G^{\widetilde{\wp}}\right)$ is the direct product of its nontrivial Sylow $p_{\lambda}$-subgroups $P_{\lambda} / G^{\widetilde{\mho}}$. The groups $P_{\lambda} / G^{\widetilde{\mho}}$ are finite, by Lemma 3.3, and, by assumption, $\Lambda$ is infinite. Each of the groups $P_{\lambda}$ is characteristic in $G$. By assumption, $G$ contains a finitely generated subgroup $H$ such that $G=H T$. Since $H \cap T$ is finite it follows that if $p_{\lambda}>|H \cap T|$ then for such $\lambda \in \Lambda, P_{\lambda} H$ is nilpotent; otherwise the chain $P_{1} H \leq P_{1} P_{2} H \leq \ldots$ would be an infinite ascending chain of subgroups all but finitely many of which are non-nilpotent, a contradiction. We write $T / G^{\widetilde{\wp}}=U / G^{\mathscr{\wp}} \times V / G^{\widetilde{\wp}}$, where each of $U / G^{\mathscr{\wp}}$ and $V / G^{\mathscr{\wp}}$ is a direct product

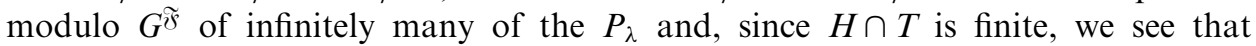
$G=H T$ is nilpotent, as in the proof of Lemma 2.7. This contradiction shows that $G / T$ is not finitely generated.

Now Lemma 3.1 implies (3), (4) and (6). Proposition 3.8 implies (2) and Proposition 3.9 implies (5). To prove (7) let $S$ be a non-nilpotent subgroup of $G$. Then $S U$ is a normal non-nilpotent subgroup of $G$ so that $G / S U$ is finitely generated, by Lemma 2.2, and divisible. Hence $G=S U$.

4. The case in which $G / G^{\mathfrak{\wp}}$ is finitely generated. In this section we require a knowledge of modules over principal ideal domains. First we gather together some of the relevant notions.

Let $J$ be a principal ideal domain, and let $\operatorname{Spec}(J)$ denote the set of all its $\max -$ imal ideals. For every ideal $P \in \operatorname{Spec} J$ we choose an element $x_{P}$ such that $P=x_{P} J$ and we let $\sigma(J)$ be the set of such elements. Then every element $y \neq 0$ has a presentation $y=u\left(x_{1}\right)^{l_{1}} \ldots\left(x_{n}\right)^{l_{n}}$, where $u \in U(J)$, the set of units of $J, x_{i} \in \sigma(J), l_{i} \in \mathbb{N}$, $1 \leq i \leq n$. This presentation is unique.

Now let $A$ be a $J$-module. Then the set $t_{J}(A)=\left\{a \in A \mid\right.$ Ann $\left._{J}(a) \neq 0\right\}$ is a submodule of $A$. We call $t_{J}(A)$ the $J$-torsion submodule of $A$. If $a \in t_{J}(A)$, then $\operatorname{Ann}_{A}(a)=y J$ and $y=u\left(x_{1}\right)^{l_{1}} \ldots\left(x_{n}\right)^{l_{n}}$, as above. Put $\pi(a)=\left\{x_{1}, \ldots, x_{n}\right\}$ and $\pi(A)=\bigcup_{a \in t_{J}(A)} \pi(a)$. Let $x \in \sigma(J)$. An element $a \in A$ is called an $x$-element if $a x^{n}=0$ for some $n \in \mathbb{N}$. The set $A_{x}$ of all $x$-elements of $A$ is a submodule. Moreover,

$$
t_{J}(A)=\bigoplus_{x \in \pi(A)} A_{x}
$$

The submodule $A_{x}$ is called the $x$-component of $A$. The module $A$ is called $J$-periodic if $A=t_{J}(A)$; the module $A$ is called $J$-torsionfree if $t_{J}(A)=0$.

In our first result we consider a natural example of a locally nilpotent group that does not satisfy max-(non-nil).

Lemma 4.1. Let $G=A \rtimes\langle g\rangle$, where $A=\operatorname{Dr}_{n \in \mathbb{N}}\left\langle a_{n}\right\rangle$ is an elementary abelian $p$-subgroup, for some prime $p$, and $g$ is an element of infinite order such that

$$
a_{1}^{g}=a_{1}, a_{n+1}^{g}=a_{n+1} a_{n}, \text { for each } n \in \mathbb{N} .
$$

Then $G$ does not satisfy max-(non-nil).

Proof. We think of $A$ as an $F_{p}\langle g\rangle$-module. Then $A$ is an $F_{p}\langle g\rangle$-periodic module; indeed, $A$ coincides with its $(g-1)$-component. Clearly, $A=[A, g]$, and so $A$ is a 
$(g-1)$-divisible module. Let $y=g^{p}$. Then $A$ is $(y-1)$-divisible also. Hence there are elements $b_{1}=a_{1}, b_{2}, \ldots, b_{n}, \ldots$ such that $b_{1}(y-1)=0, b_{n+1}(y-1)=b_{n},(n \in \mathbb{N})$. Let $B=\left\langle b_{n} \mid n \in \mathbb{N}\right\rangle$. Then $B$ is an $F_{p}\langle y\rangle$-submodule of $A$ and the subgroup $\langle B, y\rangle$ is hypercentral, but non-nilpotent. However,

$$
A=B \oplus B g \oplus \ldots \oplus B g^{p-1},
$$

and, in particular, $A / B$ does not satisfy $\max -\langle y\rangle$. The result follows from Lemma 2.3 .

Clearly if $G$ is locally nilpotent and satisfies max-(non-nil), then $G$ involves no group of the type indicated in Lemma 4.1. Now suppose that $A$ is a normal elementary abelian $p$-subgroup of $G$ and suppose that $g \in G$ has infinite order. The submodule $B$ of the $F_{p}\langle g\rangle$-module $A$ is called a basic submodule (more precisely $(g-1)$-basic) if $B$ satisfies the following conditions:

(i) $B$ is a direct sum of cyclic submodules;

(ii) $B(g-1)^{n}=B \cap A(g-1)^{n}$, for all $n \in \mathbb{N}$;

(iii) $A / B$ is $(g-1)$-divisible.

As in Abelian Group Theory (see, for example, [3, Chapter 6]) one can prove that $A$ contains a basic submodule $B$.

Lemma 4.2. Let $G$ be a group satisfying max-(non-nil) and suppose that $A$ is an elementary abelian normal p-subgroup of $G$, for some prime $p$. Suppose that $g$ is an element of infinite order and that the $F_{p}\langle g\rangle$-module $A$ coincides with its $(g-1)$-component. Then there is an integer $n$ such that $[A, \underbrace{g, \ldots, g}_{n}]=1$.

Proof. We suppose the contrary. Suppose first that there exists a natural number $n$ such that $g^{n} \in C_{G}(A)$. We claim that $\langle A, g\rangle$ is nilpotent in this case. Factoring by $g^{n}$ we only need to show that $\langle A, g\rangle /\left\langle g^{n}\right\rangle$ is nilpotent. However if $a \in A$, then $a$ has only finitely many conjugates in $\langle A, g\rangle$ and so $\langle a\rangle^{\langle g\rangle}$ is finite. We may then construct an infinite strictly ascending chain of finite subgroups $N_{1}\langle g\rangle /\left\langle g^{n}\right\rangle \supsetneqq N_{2}\langle g\rangle /\left\langle g^{n}\right\rangle \supsetneqq \ldots$ with $a \in N_{i} \leq A$. By Lemma 2.4, each $N_{i}\langle g\rangle /\left\langle g^{n}\right\rangle$ is nilpotent; if $n$ is relatively prime to $p$ this implies $\left[N_{i},\langle g\rangle\right] \leq\left\langle g^{n}\right\rangle$ for each $i$ and hence $[A, g, g]=1$, since $a$ is an arbitrary element of $A$. We may therefore assume $n$ is a power of $p$ but, in this case, $\langle A, g\rangle$ is nilpotent, by [15, Lemma 6.34]. Thus, if $\langle g\rangle \cap C_{G}(A) \neq 1$, the result follows.

Hence we may suppose that $\langle g\rangle \cap C_{G}(A)=1$. Let $B$ be a $(g-1)$-basic submodule of $A$. Clearly $A$ is infinite. Suppose that $B \neq A$. Then $A / B$ contains a nontrivial submodule $\bar{C}=\left\langle\bar{c}_{n} \mid n \in \mathbb{N}\right\rangle$ such that $\bar{c}_{1}(g-1)=0, \bar{c}_{n+1}(g-1)=\bar{c}_{n}, n \in \mathbb{N}$. However Lemma 4.1 shows that in this case $A$ does not satisfy max-(non-nil). It follows that $A=B$ and so

$$
A=\bigoplus_{\lambda \in \Lambda} A_{\lambda}
$$

where $A_{\lambda}=a_{\lambda} F_{p}\langle g\rangle$ is a cyclic submodule, for each $\lambda \in \Lambda$. Let $s_{\lambda} \in \mathbb{N}$ be such that $\operatorname{Ann}_{F_{p}\langle g\rangle}\left(a_{\lambda}\right)=(g-1)^{s_{\lambda}} F_{p}\langle g\rangle$. Our assumption shows that the set $\left\{s_{\lambda} \mid \lambda \in \Lambda\right\}$ is infinite. Hence there are subsets $\Lambda_{1}, \Lambda_{2}$ such that $\Lambda_{1} \cap \Lambda_{2}=\emptyset, \Lambda_{1} \cup \Lambda_{2}=\Lambda$ and both sets $\left\{s_{\lambda} \mid \lambda \in \Lambda_{1}\right\}$ and $\left\{s_{\lambda} \mid \lambda \in \Lambda_{2}\right\}$ are infinite. Put 


$$
E_{i}=\bigoplus_{\lambda \in \Lambda_{i}} A_{\lambda}, \text { for } i=1,2
$$

Then for $i=1,2,\left\langle E_{i}, g\right\rangle$ is non-nilpotent. In particular, Lemma 2.3 implies that $A / E_{1} \cong E_{2}$ satisfies max- $\langle g\rangle$, which gives a contradiction since $\left|\Lambda_{2}\right|$ is infinite. Hence there is an integer $n$ such that $A_{\lambda}(g-1)^{n}=0$, for every $\lambda \in \Lambda$. Hence $A(g-1)^{n}=0$ and the result follows.

Lemma 4.3. Let $G$ be a locally nilpotent group satifying max-(non-nil) and suppose that $A$ is a normal elementary abelian p-subgroup, for some prime $p$. If $G / C_{G}(A)$ is finitely generated, then there is an integer $n$ such that $\left[A,_{n} G\right]=1$.

Proof. If $A$ is finite the result is clear. We suppose that $A$ is infinite. Let $T / C_{G}(A)$ be the (finite) torsion subgroup of $G / C_{G}(A)$ and let $G / T$ have the ascending central series

$$
T=Z_{0} \leq Z_{1} \leq \ldots \leq Z_{m}=G
$$

such that $Z_{i+1} / Z_{i}=\left\langle g_{i+1} Z_{i}\right\rangle$ is an infinite cyclic group, for $0 \leq i \leq m-1$. Since $T / C_{G}(A)$ is finite, $A$ has a finite upper $T$-central series

$$
1=A_{0} \leq A_{1} \leq \ldots \leq A_{n_{1}}=A
$$

(see, for example, $\left[\mathbf{1 5}\right.$, Lemma 6.34]) and, in particular, $\left[A,,_{n_{1}} T\right]=1$. Thus we may suppose that $G \neq T$. Since $T$ is normal in $G$ the subgroups $A_{i}$ are $G$-invariant. Let $a A_{i} \in A_{i+1} / A_{i}$ and $B / A_{i}=\left\langle a A_{i}\right\rangle^{\left\langle g_{1}\right\rangle}$. Since $\left\langle a, g_{1}\right\rangle$ is nilpotent, $B / A_{i}$ is finite. Thus it has a finite $\left\langle g_{1}\right\rangle$-central series and it follows that $A_{i+1} / A_{i}$ coincides with its $\left(g_{1}-1\right)$ component. By Lemma 4.2 there is an integer $n_{2, i}$ such that $\left[A_{i+1}, n_{2, i} g_{1}\right] \leq A_{i}$, for $1 \leq i \leq n_{1}$. Let $n_{2}$ be the sum of the $n_{2, i}$. Then $\left[A,,_{n_{2}} Z_{1}\right]=1$. Similar arguments imply that there is an integer $n$ such that $\left[A,{ }_{n} G\right]=1$.

As an immediate corollary we have the following result.

COROLlARY 4.4. Let $G$ be a locally nilpotent group satisfying max-(non-nil) and suppose that $A$ is a normal elementary abelian p-subgroup, for some prime $p$. Suppose that $G / A$ is finitely generated. Then $G$ is nilpotent.

We can put this information to work to obtain some further information concerning the finite residual.

Lemma 4.5. Let $G$ be a locally nilpotent group satisfying max-(non-nil). Let $G^{\widetilde{\Im}}$ be the finite residual of $G$ and suppose that $G / G^{\mathfrak{\Im}}$ is finitely generated. If $G$ is nonnilpotent, then $G^{\mathfrak{\wp}}$ is $\mathfrak{\lessgtr}$-perfect.

Proof. Let $R=G^{\widetilde{S}}$ and suppose that $R$ contains a proper subgroup of finite index. Then there is a prime $p$ such that $R$ contains a subgroup $H$ of index $p$. Put $U=$ core $_{G} H$; then $R / U$ is an elementary abelian $p$-group. By Corollary $4.4, G / U$ is nilpotent. By Proposition 2 of [5], $G$ contains a normal subgroup $V \geq U$ such that $V / U$ is torsionfree and $G / V$ is a bounded $p$-group. Thus $V \cap R=U$. If $G / V$ is finite, 
then $R / U=R /(V \cap R) \cong R V / V$ is also finite, so that $G / U$ is finitely generated and hence residually finite. This contradicts the fact that $R=G^{\widetilde{\mho}}$. Hence $G / V$ is infinite. Since $G / V$ is nilpotent, $(G / V) /(G / V)^{\prime}$ is infinite by [15, Corollary to Theorem 2.26]. It follows from Corollary 2.6 that $G$ is nilpotent. This contradiction shows that $R$ is $\mathfrak{\Im}$-perfect.

Our next few results show that periodic normal abelian subgroups of our group $G$ are quite restricted, at least when an element of infinite order does not act nilpotently.

Lemma 4.6. Let $G$ be a locally nilpotent group satisfying max-(non-nil). Let $A$ be a normal abelian p-subgroup, for some prime $p$, and suppose that $g \in G$ is an element of infinite order. Suppose that $A=[A, g]$. Then $C_{A}(g)$ is not divisible.

Proof. For every element $a \in A$ the subgroup $\langle a, g\rangle$ is nilpotent. It follows that $C_{A}(g) \neq 1$ and it is easy to see that $\langle A, g\rangle$ is hypercentral. Thus $A$ has upper $\langle g\rangle-$ central series

$$
1=C_{0} \leq C_{1} \leq \ldots C_{\alpha} \leq C_{\alpha+1} \leq \ldots C_{\gamma}=A
$$

where $C_{1}=C_{A}(g)$ and $C_{\alpha+1} / C_{\alpha}=C_{A / C_{\alpha}}(g)$ for $\alpha<\gamma$. Since $\langle a, g\rangle$ is nilpotent there is an integer $n$ such that $a \in C_{n}$. Hence $A=\bigcup_{n \in \mathbb{N}} C_{n}$, and $A=C_{\omega}$. Assume that $C_{1}$ is divisible. Since $A=[A, g],\left[C_{n+1}, g\right]=C_{n}$, for each $n \in \mathbb{N}$, and the map

$$
\phi: a \longmapsto[a, g], a \in C_{n+1},
$$

from $C_{n+1}$ onto $C_{n}$ is an epimorphism with kernel $C_{1}$. Thus if $C_{n}$ is divisible, then so is $C_{n+1}$ and it follows by induction on $n$ that $C_{n}$ is divisible, for all $n \in \mathbb{N}$. Now $C_{n} \neq A$, for any $n \in \mathbb{N}$, since $A=[A, g]$. We have $C_{2}=C_{1} \times D_{1}$, for some divisible subgroup $D_{1}$. Since $\left[C_{2}, g\right]=C_{1}$ we have $\left[D_{1}, g\right]=C_{1}$. Let $c_{1} \in \Omega_{1}\left(C_{1}\right)$. Then there is an element $c_{2} \in D_{1}$ such that $c_{1}=\left[c_{2}, g\right]$. Suppose that $\left|c_{2}\right|>p$. Then $c_{2}^{p} \neq 1$ and $1=c_{1}^{p}=\left[c_{2}, g\right]^{p}=\left[c_{2}^{p}, g\right]$ so that $1 \neq c_{2}^{p} \in C_{A}(g)=C_{1}$. But $c_{2}^{p} \in D_{1}$. This contradiction shows that $\left|c_{2}\right|=p$. Inductively we can construct a set of elements $\left\{c_{n} \mid n \in \mathbb{N}\right\}$ such that $\left\langle c_{n} \mid n \in \mathbb{N}\right\rangle=\bigoplus_{n \in \mathbb{N}}\left\langle c_{n}\right\rangle,\left|c_{n}\right|=p,\left[c_{1}, g\right]=1$ and $\left[c_{n+1}, g\right]=c_{n}$, for all $n \in \mathbb{N}$. Lemma 4.1 now gives a contradiction. Hence $C_{1}=C_{A}(g)$ is not divisible.

COROLlary 4.7. Let $G$ be a locally nilpotent group satisfying max-(non-nil). Let $A$ be a normal abelian p-subgroup, for some prime $p$, and suppose that $g \in G$ is an element of infinite order. Assume that $A=[A, g]$. Then $C_{A}(g)$ contains no non-trivial divisible subgroups.

Proof. We may as well suppose that $G=\langle A, g\rangle$. Suppose, by way of contradiction, that $D$ is a non-trivial divisible subgroup of $C_{A}(g)$. Then there exists a subgroup $E$ such that $C_{A}(g)=D \times E$. Clearly, $E$ is $\langle g\rangle$-invariant. Let $U$ be a maximal $\langle g\rangle$-invariant subgroup of $A$ such that $E \leq U$ and $D \cap U=1$. Let $C / U=C_{A / U}(g)$. Since $D U / U$ is divisible there is a subgroup $F$ of $C$ such that $C / U=D U / U \times F / U$. Then $C=D F$ and $[F, g] \leq U \leq F$ so that $F$ is $\langle g\rangle$-invariant. However $D \cap F \leq D \cap U=1$ and so the maximal choice of $U$ yields $F=U$. Hence $C / U=D U / U$ is a divisible group. We thus obtain a contradiction on applying Lemma 4.6 to $\langle A / U, g U\rangle$. 
COROLlary 4.8. Let $G$ be a locally nilpotent group satisfying max-(non-nil). Let $A$ be a normal abelian p-subgroup, for some prime $p$, and suppose that $g \in G$ is an element of infinite order. Assume that $A=[A, g]$. Then $C_{A}(g)$ is a bounded subgroup.

Proof. Suppose, on the contrary, that $C=C_{A}(g)$ is not bounded. Let $B$ be a basic subgroup of $C$. If $B \neq C$, then $C / B$ is a divisible group. Thus $C_{A / B}(g)$ contains a divisible subgroup, which is impossible by Corollary 4.7. Thus $B=C$. Since $C$ is not bounded, $C$ contains a subgroup $E=\operatorname{Dr}_{n \in \mathbb{N}}\left\langle e_{n}\right\rangle$ such that $\left|e_{n}\right|=p^{n}$, for $n \in \mathbb{N}$. Let $U=\left\langle\left(e_{n}\right)\left(e_{n+1}\right)^{-p} \mid n \in \mathbb{N}\right\rangle$. Now $E / U$ is a Prüfer $p$-group. Hence $C_{A / U}(g)$ contains a divisible subgroup. This contradiction shows that $C_{A}(g)$ is bounded.

COROLlary 4.9. Let $G$ be a locally nilpotent group satisfying max-(non-nil). Let $A$ be a normal abelian p-subgroup, for some prime $p$, and suppose that $g \in G$ is an element of infinite order. Assume that $A=[A, g]$. If $B$ is a $\langle g\rangle$-invariant subgroup of $A$ such that $\langle B, g\rangle$ is nilpotent, then $B$ is bounded.

Proof. Clearly $\langle A, g\rangle$ is hypercentral. Let

$$
1=C_{0} \leq C_{1} \leq \ldots \leq C_{n} \leq \ldots C_{\omega}=A
$$

be the upper $\langle g\rangle$-central series of $A$. Since $\left[C_{n+1}, g\right]=C_{n}$ and $C_{n+1} / C_{1} \cong C_{n}$, it follows from Corollary 4.8 and induction on $n$ that $C_{n}$ is bounded, for every $n \in \mathbb{N}$. Let

$$
1=B_{0} \leq B_{1} \leq \ldots \leq B_{m}=B
$$

be the upper $\langle g\rangle$-central series of $B$. Clearly $B_{i} \leq C_{i}$ for all $i \in \mathbb{N}$ and in particular, $B=B_{m} \leq C_{m}$. Hence $B$ is bounded.

Lemma 4.10. Let $G$ be a locally nilpotent group satisfying max-(non-nil). Let $A$ be a normal abelian p-subgroup, for some prime $p$, and suppose that $g \in G$ is an element of infinite order. If $A=[A, g]$, then $A$ is a Cernikov subgroup.

Proof. Let $C_{1}=C_{A}(g)$. By Corollary 4.8, $C_{1}$ is bounded and so $C_{1}=\operatorname{Dr}_{\lambda \in \Lambda}\left\langle c_{\lambda}\right\rangle$, for some index set $\Lambda$. Choose an index $\lambda_{0}$ and let $a=c_{\lambda_{0}}$. Then $C_{1}=\langle a\rangle \times B_{1}$ where $B_{1}=\operatorname{Dr}_{\lambda \neq \lambda_{0}}\left\langle c_{\lambda}\right\rangle$, and the subgroup $B_{1}$ is $\langle g\rangle$-invariant. Let $M$ be a $\langle g\rangle$-invariant subgroup of $A$, maximal subject to $B_{1} \leq M$ and $M \cap\langle a\rangle=1$. Put $C_{2} / M=C_{A / M}(g)$. If $X / M$ is a non-trivial subgroup of $C_{2} / M$, then $\left[C_{2}, g\right] \leq M$ implies $[X, g] \leq M \leq X$ and so $X$ is $\langle g\rangle$-invariant. It follows from the choice of $M$ that every non-trivial subgroup of $C_{2} / M$ has non-trivial intersection with $\langle a M\rangle$. Hence $C_{2} / M$ is finite, since $C_{2} / M$ is bounded, by Corollary 4.8. In particular, $C_{2} / M$ is an Artinian $\mathbb{Z}\langle g\rangle$ module. Since $A / M$ has its upper $\langle g\rangle$-central series of length $\omega$, Lemma 1 of [18] implies that $A / M$ is an Artinian $\mathbb{Z}\langle g\rangle$-module. If $g^{n} \in C_{G / M}(A / M)$ for some natural number $n$, then the subgroup $\langle A / M, g M\rangle$ is a locally nilpotent group satisfying min- $n$ and hence is Cernikov. Suppose then that $\langle g M\rangle \cap C_{G / M}(A / M)=1$ and that $A / M$ is not Černikov. Then, by [11, Theorem 1], $A$ contains $\langle g\rangle$-invariant subgroups $U, V$ satisfying the following conditions: $M \leq V \leq U, V / M$ is a Cernikov group, $U / V=\operatorname{Dr}_{n \in \mathbb{N}}\left\langle u_{n} V\right\rangle$ is an elementary abelian p-group and $\left[u_{1} V, g\right]=1$, $\left[u_{n+1} V, g\right]=u_{n} V$, for all $n \in \mathbb{N}$. This contradicts Lemma 4.1 . 
Hence $A / M$ is a Černikov group, in any case. Since $A / M=[A / M, g], A / M$ is infinite and therefore $A / M$ does not satisfy the maximal condition for $\langle g\rangle$-invariant subgroups. By Lemma 2.3, it follows that $\langle M, g\rangle$ is a nilpotent subgroup. By Corollary 4.9, $M$ is bounded. Using a corollary to Lemma 1 of [8], we can write $A=D \times E$, where $D$ is a divisible Cernikov subgroup and $E$ is a bounded subgroup. Clearly, $D$ is $\langle g\rangle$-invariant. Since $A / D$ is bounded it has a finite series of $\langle g\rangle$-invariant subgroups with elementary abelian $p$-factors. Lemma 4.2 then implies that $\langle A / D, g D\rangle$ is nilpotent. Hence $[A / D, g] \neq A / D$, which is a contradiction unless $A=D$. Thus $A$ is a Černikov group.

Lemma 4.11. Let $G$ be a locally nilpotent group satisfying max-(non-nil). Let $A$ be a normal abelian p-subgroup of $G$, for some prime $p$, and suppose that $g \in G$ is an element of infinite order. Let $A_{0}=A$ and $A_{n+1}=\left[A_{n}, g\right]$, for all $n \in \mathbb{N}$. Then there is an integer $m$ such that $A_{m}=A_{m+1}$.

Proof. Suppose, on the contrary, that $A_{n+1} \neq A_{n}$ for all $n \in \mathbb{N}$. Suppose first that $A_{\omega}=\bigcap_{n \in \mathbb{N}} A_{n}=1$. For each $a \in A,\langle a\rangle^{\langle g\rangle}$ is finite, since $\langle a, g\rangle$ is nilpotent.

Let $1 \neq d_{1} \in A, D_{1}=\left\langle d_{1}\right\rangle^{\langle g\rangle}$. Since $D_{1}$ is finite, from $\bigcap_{n \in \mathbb{N}} A_{n}=1$, we see that there is an integer $k_{1}$ such that $D_{1} \cap A_{k_{1}}=1$. Since $\left\langle D_{1}, g\right\rangle=\left\langle d_{1}, g\right\rangle$ is nilpotent there is an integer $m_{1}$ such that $[D_{1}, \underbrace{g, \ldots, g]}_{m_{1}}=\left[D_{1}, m_{1} g\right]=1$. If there is an integer $r$ such that $\left[A_{k_{1}}, r g\right]=1$, then $A_{k_{1}+r}=1$. Hence there is an element $d_{2} \in A_{k_{1}}$ and an integer $m_{2}>m_{1}$ such that $\left[d_{2}, m_{2} g\right]=1$ and $\left[d_{2}, m_{2}-1 g\right] \neq 1$.

Set $D_{2}=\left\langle d_{2}\right\rangle^{\langle g\rangle}$ so that $D_{2}$ is again finite. Clearly $D_{2} \leq A_{k_{1}}$ and, in particular, $D_{1} \cap D_{2}=1$. The subgroup $D_{1} D_{2}$ is finite and so there exists an integer $k_{2}$ such that $D_{1} D_{2} \cap A_{k_{2}}=1$. In this way we constuct an infinite family of finite $\langle g\rangle$-invariant subgroups $\left\{D_{n} \mid n \in \mathbb{N}\right\}$ and integers $\left\{m_{n} \mid n \in \mathbb{N}\right\}$ such that

$$
\left\langle D_{n} \mid n \in \mathbb{N}\right\rangle=\operatorname{Dr}_{n \in N} D_{n} \text {, and }\left[D_{i}, m_{i} g\right]=1 \text { but }\left[D_{i}, m_{i}-1 g\right] \neq 1
$$

and

$$
m_{1}<m_{2}<\ldots<m_{n}<\ldots
$$

The subgroup $\left\langle g, D_{n} \mid n \in \mathbb{N}\right\rangle$ is clearly non-nilpotent, a contradiction to Lemma 2.7.

Finally, if $A_{\omega} \neq 1$ we apply the argument above to $A / A_{\omega}$ and obtain the result in general.

COROLlary 4.12. Let $G$ be a locally nilpotent group satisfying max-(non-nil). Let $A$ be a normal abelian p-subgroup of $G$, for some prime $p$, and suppose that $g \in G$ is an element of infinite order. Then either $\langle A, g\rangle$ is nilpotent or A contains a divisible Cernikov subgroup $D$ such that $D=[D, g]$ and $A / D$ is finite.

Proof. Let $A_{0}=A$, and $A_{n+1}=\left[A_{n}, g\right]=\left[A,,_{n+1} g\right]$, for all $n \in \mathbb{N}$. By Lemma 4.11 there is an integer $m$ such that $A_{m+1}=A_{m}$. If $A_{m}=1$, then $\langle A, g\rangle$ is nilpotent. Suppose that $D=A_{m} \neq 1$. Then $D=[D, g]$, and by Lemma $4.10, D$ is a Černikov group. Clearly $D$ is divisible and $\langle D, g\rangle$ is non-nilpotent. By Lemma $2.3, A / D$ satisfies max- $\langle g\rangle$ and, since $\langle A, g\rangle$ is hypercentral, it follows that $A / D$ is finite. 
COROLlary 4.13. Let $G$ be a locally nilpotent group satisfying max-(non-nil). Let $A$ be a normal abelian periodic subgroup of $G$ and suppose that $g \in G$ is an element of infinite order. Then either $\langle A, g\rangle$ is nilpotent or A contains a divisible Cernikov subgroup $D$ such that $D=[D, g]$ and $A / D$ is finite.

Proof. If the set $\pi(A)$ is infinite, then $\langle A, g\rangle$ is nilpotent, by Lemma 2.7. Suppose that $\pi(A)$ is finite, let $p \in \pi(A)$ and let $A_{p}$ be a Sylow $p$-subgroup of $A$. If $\left\langle A_{p}, g\right\rangle$ is nilpotent, for every $p \in \pi(A)$, then $\langle A, g\rangle$ is also nilpotent. Suppose that $\left\langle A_{p}, g\right\rangle$ is non-nilpotent for some prime $p$. Then Corollary 4.12 yields that $A_{p}$ contains a divisible Černikov subgroup $D$ such that $D=[D, g]$. In particular $\langle D, g\rangle$ is non-nilpotent. By Lemma $2.3, A / D$ satisfies $\max -\langle g\rangle$. Hence $A / D$ is finite since $\langle A, g\rangle$ is hypercentral.

Let $G$ be a group and $A$ a divisible normal subgroup of $G$. We say that $A$ is divisibly irreducible in $G$ if $A$ contains no proper $G$-invariant divisible subgroups.

Lemma 4.14. Let $G$ be a group and $A$ a normal abelian subgroup of $G$ satisfying the following conditions:

(i) $G / C_{G}(A)$ is nilpotent;

(ii) $A \cap Z(G)$ contains a subgroup $B$ such that $A / B$ is a divisible Cernikov group;

(iii) $A / B$ is divisibly irreducible in $G$;

(iv) $A / B=[A / B, G]$.

Then $A$ contains a G-invariant divisible Černikov subgroup $D$ such that $D=[D, G], D$ is divisibly irreducible in $G, A=B D$ and $B \cap D$ is finite.

Proof. Let $C_{G}(A) \neq z C_{G}(A) \in Z\left(G / C_{G}(A)\right)$. Then the mapping $\phi: a \mathrm{\imath} \longrightarrow[a, z]$, $(a \in A)$, is a $\mathbb{Z} G$-endomorphism of $A$. Thus $K=\operatorname{ker} \phi$ and $D=\operatorname{Im} \phi$ are $G$-invariant subgroups of $A$ and, since $B \leq Z(G)$, we have $B \leq K$. It follows that either $K / B$ is finite or $K=A$, since $A / B$ is divisibly irreducible in $G$. However, by choice of $z$, $K \neq A$. Hence $K / B$ is finite. It follows that $D$ is a divisible Cernikov subgroup, $D$ is divisibly irreducible and either $D=[D, G]$ or $[D, G]=1$. In the latter case $D=[A, z]$ is central in $G$ and so $[A, z, G]=1$. By definition of $z$ we also have $[z, G, A]=1$ and hence $[G, A, z]=1$. However (ii) and (iv) imply $1 \neq D=[A, z]=[[G, A] B, z]=$ $[G, A, z]$. This contradiction implies $D=[D, G]$. Thus $D \cap B$ is finite and $D B / B \cong D /(D \cap B)$ is an infinite $G$-invariant divisible subgroup of $A / B$, so that $B D=A$.

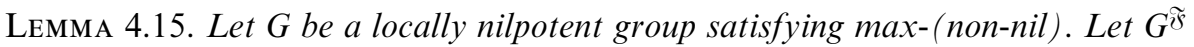
be the finite residual of $G$. Suppose that $G / G^{\mathfrak{\wp}}$ is finitely generated and $G^{\Re}$ is not Cernikov. If $G^{\mathfrak{\mho}}$ is nilpotent, then $G$ is nilpotent.

Proof. Suppose, on the contrary, that $G$ is non-nilpotent. By Lemma 4.5, $R=G^{\mathfrak{\mho}}$ is $\mathfrak{\wp}$-perfect. Since $R$ is periodic, by Corollary 3.2, and nilpotent, then $R$ is a divisible abelian subgroup. (See, for example, [15, Corollary 2 of Theorem 9.23].) Let $T / R$ be the torsion subgroup of $G / R$. Then $G / T$ is a finitely generated torsionfree nilpotent group and so $G / T$ has a central series

$$
T=C_{0} \leq C_{1} \leq \ldots \leq C_{m}=G
$$


such that $C_{i+1} / C_{i}=\left\langle g_{i+1} C_{i}\right\rangle$, for $0 \leq i \leq m-1$. Let $P$ be a Prüfer $p$-subgroup of $R$, and let $U=P^{T}$. Since $T / R$ is finite, $U$ is a divisible Černikov group. Since $R$ is not Cernikov, $R=U \times V$ for some non-trivial subgroup $V$. By Theorem 2.7 of [2], there exists a $T$-invariant subgroup $W$ such that $R=U W$ and $U \cap W$ is bounded. Since $T / R$ is finite there is a finite subgroup $F$ such that $T=F R$. Since the factor groups $R / U$ and $R / W$ are both divisible, they do not satisfy max- $F$. By Lemma 2.3, $U F$ and $W F$ are both nilpotent and so there is an integer $k$ such that $\left[U,_{k} T\right]=\left[W,_{k} T\right]=1$. Since $R=U W$, we see that $\left[R,{ }_{k} T\right]=1$ and hence $T$ is nilpotent. Thus we may assume that $T \neq G$. Since $R$ is divisible, $R \leq Z(T)$ by [15, Lemma 3.29.1]) and $\left\langle R, g_{1}\right\rangle$ is nilpotent.

Let

$$
1=R_{0} \leq R_{1} \leq \ldots \leq R_{l_{1}}=R
$$

be the upper $\left\langle g_{1}\right\rangle$-central series of $R$. By Corollary 4.13, there is an integer $l_{2}$ such that $\left[R, l_{2} g_{2}\right]=1$. It follows that $\left[R, l_{1} l_{2} C_{2}\right]=1$. Using similar arguments and a simple induction we see that the group $G$ is nilpotent, a contradiction, which proves the result.

Theorem B. Let $G$ be a locally nilpotent group satisfying max-(non-nil). Let $G^{\Re}$ be the finite residual of $G$ and suppose that $G / G^{\Re}$ is finitely generated. If $G$ is nonnilpotent and $G^{\widetilde{\mho}}$ is nilpotent, then $G^{\mathfrak{\mho}}$ is a divisible Cernikov group. Moreover, $G^{\mathfrak{\mho}}$ is divisibly irreducible and $\left[G^{\widetilde{\Im}}, G\right]=G^{\widetilde{\Im}}$.

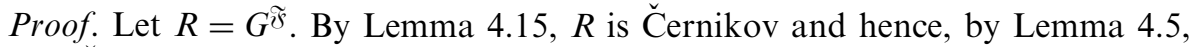
divisible Cernikov. Let $A$ be a minimal $G$-invariant divisible subgroup of $R$ and set $A_{1}=[A, G]$. Since $[A, G]$ is a divisible $G$-invariant subgroup of $A$ we have $A_{1}=A$ or $A_{1}$ is trivial. Since $R$ is a Černikov group it has a finite series of $G$-invariant subgroups

$$
1=R_{0} \leq R_{1} \leq \ldots \leq R_{t}=R
$$

every factor of which is divisibly irreducible. In particular, either $\left[R_{i+1}, G\right] \leq R_{i}$ or $\left[R_{i+1} / R_{i}, G\right]=R_{i+1} / R_{i}$, for each $i$, with $0 \leq i \leq t-1$. Since $G$ is non-nilpotent there exists an integer $l$ such that $\left[R_{l+1} / R_{l}, G\right]=R_{l+1} / R_{l}$. Let $l$ be the least integer with this property. Suppose that $l>0$. By Lemma $4.14, R_{l+1} / R_{l-1}$ contains a $G$-invariant divisible Cernikov subgroup $Q_{l} / R_{l-1}$ such that $R_{l+1} / R_{l-1}=\left(R_{l} / R_{l-1}\right)\left(Q_{l} / R_{l-1}\right)$, $Q_{l} / R_{l-1}$ is divisibly irreducible and $\left[Q_{l} / R_{l-1}, G\right]=Q_{l} / R_{l-1}$. Using similar arguments we see that $R$ contains a $G$-invariant divisibly irreducible subgroup $D$ such that $[D, G]=D$. Since $G / R$ is finitely generated, $G=R F$ for some finitely generated subgroup $F$. Then $\langle D, F\rangle$ is non-nilpotent, and Lemma 2.3 implies that $R / D$ satisfies max $-F$. Since $R / D$ is divisible and periodic, $R / D$ is finite. Hence $R=D$ and the result follows.

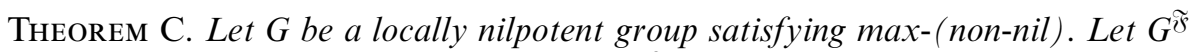
be the finite residual of $G$ and suppose that $G / G^{\mathfrak{\wp}}$ is finitely generated. Let $G$ be nonnilpotent and non-minimax.

(i) $G^{\widetilde{\mho}}$ is periodic.

(ii) $G^{\mathfrak{\wp}}$ is $\mathfrak{s}$-perfect. 
(iii) $G^{\widetilde{\wp}}$ is non-nilpotent and every proper subgroup of $G^{\Re}$ is nilpotent. Furthermore $G^{\widetilde{\mho}}$ is nilpotent-by-Černikov and $G$ is soluble.

In particular, $G^{\mathfrak{\mho}}$ is a p-group, for some prime $p$, having an ascending series of $G$ invariant subgroups

$$
1=A_{0} \leq A_{1} \leq \ldots \leq A_{n} \leq \ldots \text { such that } \bigcup_{n \in \mathbb{N}} A_{n}=G^{\widetilde{\mho}}
$$

and such that every subgroup $A_{n}$ is nilpotent.

Proof. Corollary 3.2 implies (i) and Lemma 4.5 implies (ii). By Theorem B, $G^{\Im}$ is not nilpotent. If $H$ is a non-nilpotent normal subgroup of $G$, then $G / H$ satisfies $\max$ by Lemma 2.2. In particular $G / H$ is residually finite and so $G^{\Re} \leq H$. Thus the proper $G$-invariant subgroups of $G^{\mathfrak{\wp}}$ are nilpotent and hence $G^{\mathfrak{\wp}}$ is a $p$-group, for some prime $p$, by Corollary 2.6 and Lemma 3.3. Thus $G^{\widetilde{\wp}}$ is a locally nilpotent $\mathfrak{\wp}$ perfect $p$-group and if $H_{i}$ is a non-nilpotent subgroup of $G^{\widetilde{\mho}}$, for some natural number $i$, then $\left|G^{\widetilde{\mho}}: H_{i}\right|$ is infinite. Since a maximal subgroup of a locally nilpotent group is normal and of finite index, $H_{i}$ is not maximal in $G^{\widetilde{\mho}}$ and so there is a proper subgroup $H_{i+1}$ of $G^{\widetilde{\mho}}$ properly containing $H_{i}$. Since $H_{i+1}$ is not nilpotent either, this argument can be repeated and, in this way, we construct an infinite ascending chain of non-nilpotent subgroups of $G^{\mathfrak{\Im}}$, contrary to the condition max-(non-nil). Hence every proper subgroup of $G^{\widetilde{\mho}}$ is nilpotent. A recent result of Asar [1] now shows that $G^{\widetilde{\mho}}$ is nilpotent-by-Cernikov and the result follows.

\section{REFERENCES}

1. A. O. Asar, Locally nilpotent $p$-groups whose proper subgroups are hypercentral-byChernikov J. London Math. Soc. (2) 61 (2000), 412-422.

2. S. Franciosi, F. de Giovanni and L. A. Kurdachenko, On groups with many almost normal subgroups, Ann. Mat. Pura Appl. 169 (1995), 35-65.

3. L. Fuchs, Infinite abelian groups (Academic Press, 1970/73). $11-15$.

4. B. Hartley, A note on the normalizer condition, Proc. Cambridge Phil. Soc. 74 (1973),

5. H. Heineken and L. A. Kurdachenko, Groups with subnormality for all subgroups that are not finitely generated, Ann. Mat. Pura Appl. 169 (1995), 203-232.

6. H. Heineken and I. J. Mohamed, A group with trivial centre satisfying the normalizer condition, J. Algebra 10 (1968), 368-376.

7. N. V. Kalashnikova and L. A. Kurdachenko, Groups which are dual to layer-finite, in Infinite groups 1994 (Ravello 1994) (Walter de Gruyter, Berlin, 1996), 103-109.

8. L. A. Kurdachenko, FC-groups with bounded orders of the elements of periodic part, Siberian Math. J. 16 (1975), 1205-1213.

9. L. A. Kurdachenko, Some conditions for embeddability of an FC - group in a direct product of finite groups and a torsion-free abelian group, Mat. Sbornik 114 (1981), 566-582; English transl. in Math. Sbornik 42 (1982), 499-514.

10. L. A. Kurdachenko, Locally nilpotent groups with the weak minimal condition for normal subgroups, Sibirsk Mat. Zh. 25 (1984), 99-106.

11. L. A. Kurdachenko and A. V. Tushev, On some classes of groups with the weak minimal condition for normal subgroups, Ukrain. Mat. Zh. 37 (1985), 457-462; English transl. in Ukraïn. Math. J. 37 (1985), 364-368.

12. L. A. Kurdachenko and D. I. Zaicev, Groups with the maximum condition for nonabelian subgroups, Ukrain Mat. Zh. 43 (1991), 925-930; English transl. in Ukrain. Math. J. 43 (1991), 863-868. 
13. F. Menegazzo, Groups of Heineken-Mohamed, J. Algebra 171 (1995), 807-825.

14. M. F. Newman and J. Wiegold, Groups with many nilpotent subgroups, Arch. Math. 15 (1964), 241-250.

15. D. J. S. Robinson, Finiteness conditions and generalized soluble groups. Vols. 1 and 2 , Ergebnisse der Mathematik und ihrer Grenzgebiete. Band 62 and 63 (Springer-Verlag, 1972).

16. O. Yu. Schmidt, Groups all of whose subgroups are nilpotent, Mat. Sbornik 31 (1924), 366-372.

17. H. Smith, Groups with few non-nilpotent subgroups, Glasgow Math. J. 39 (1997), $141-151$

18. D. I. Zaicev, On the properties of groups inherited by their normal subgroups, Ukraï. Mat. Zh. 38 (1986), 707-713; English transl. in Ukraï. Math. J. 38 (1986), 593-598. 